\title{
Ultra-high resolution Fourier domain optical coherence tomography for resolving thin layers in painted works of art
}

\author{
C. S. Cheung, Haida Liang* \\ School of Science and Technology, Nottingham Trent University, Nottingham NG11 8NS, UK \\ *haida.liang@ntu.ac.uk
}

\begin{abstract}
While OCT has been applied to the non-invasive examination of the stratigraphy of paint layers in recent years, it has been recognized that the resolutions of commercially available OCT cannot compete in depth resolution with conventional microscopic examination of cross-sections of paint samples. It is necessary to achieve resolutions better than 3 microns to resolve the thinnest layers of paint and varnish. In this paper, we demonstrate a Fourier domain ultrahigh resolution OCT at $810 \mathrm{~nm}$ with depth resolution of $1.8 \mu \mathrm{m}$ in air (or $1.2 \mu \mathrm{m}$ in varnish or paint).
\end{abstract}

Keywords: Optical Coherence Tomography, Fourier domain, ultra-high resolution, micron-scale

\section{INTRODUCTION}

Scientific instruments for use in the area of art history, conservation, archaeology and heritage science have been developing rapidly. Examination of works of art is essential for conservation, preservation and understanding of material change and Optical Coherence Tomography (OCT) has proved invaluable in this field.

Optical Coherence Tomography (OCT) is a non-invasive, non-contact 3D imaging technique that uses the coherent properties of broad band light sources, making the technique a very useful diagnostic tool for visualization of subsurface microstructure. OCT has found applications in the examination of jade, ceramics, ancient glass, enamel, parchment and faience $[1,2,3,4]$. It has also been used for dynamic monitoring of the wetting and drying of different varnishes, varnish removal using solvents, real time laser ablation of varnish layers and tracking of canvas deformation due to environmental changes $[1,2,5]$. Besides the visualization of the stratigraphy of paint and varnish layers, application of OCT to paintings has shown to be the most sensitive technique for revealing preparatory drawings beneath paint layers owing to its high dynamic range and depth selection capabilities $[2,6]$.

However, the depth resolution of OCTs used in these applications still cannot match those of microscopic examination of sampled paint cross-sections. It is known that some varnish and paint layers can be as thin as $\sim 3$ microns. The depth resolution of commercial OCT systems is rarely better than $6 \mu \mathrm{m}$ in air. A depth resolution of $<3 \mu \mathrm{m}$ is needed to resolve the thinnest varnish and paint layers. Since the depth resolution of OCT is proportional to the source bandwidth, ultra wide bandwidth light allows a greater depth resolution to be achieved.

While full field time domain OCT in the visible part of the spectrum can reach depth resolutions of $\sim 1 \mu \mathrm{m}[7,8]$, they have very limited depth penetration as most paints are opaque in the visible range. Such instruments will be restricted to examining mostly the top varnish layers. In addition, time domain instruments are less sensitive than Fourier domain instruments.

Taking advantage of the established development of supercontinuum generated light (NKT SuperK Versa), a broadband continuous spectra of light with FWHM bandwidth of $180 \mathrm{~nm}$ is used in a spectral domain OCT system to achieve a high depth resolution imaging. 


\section{INSTRUMENT SETUP}

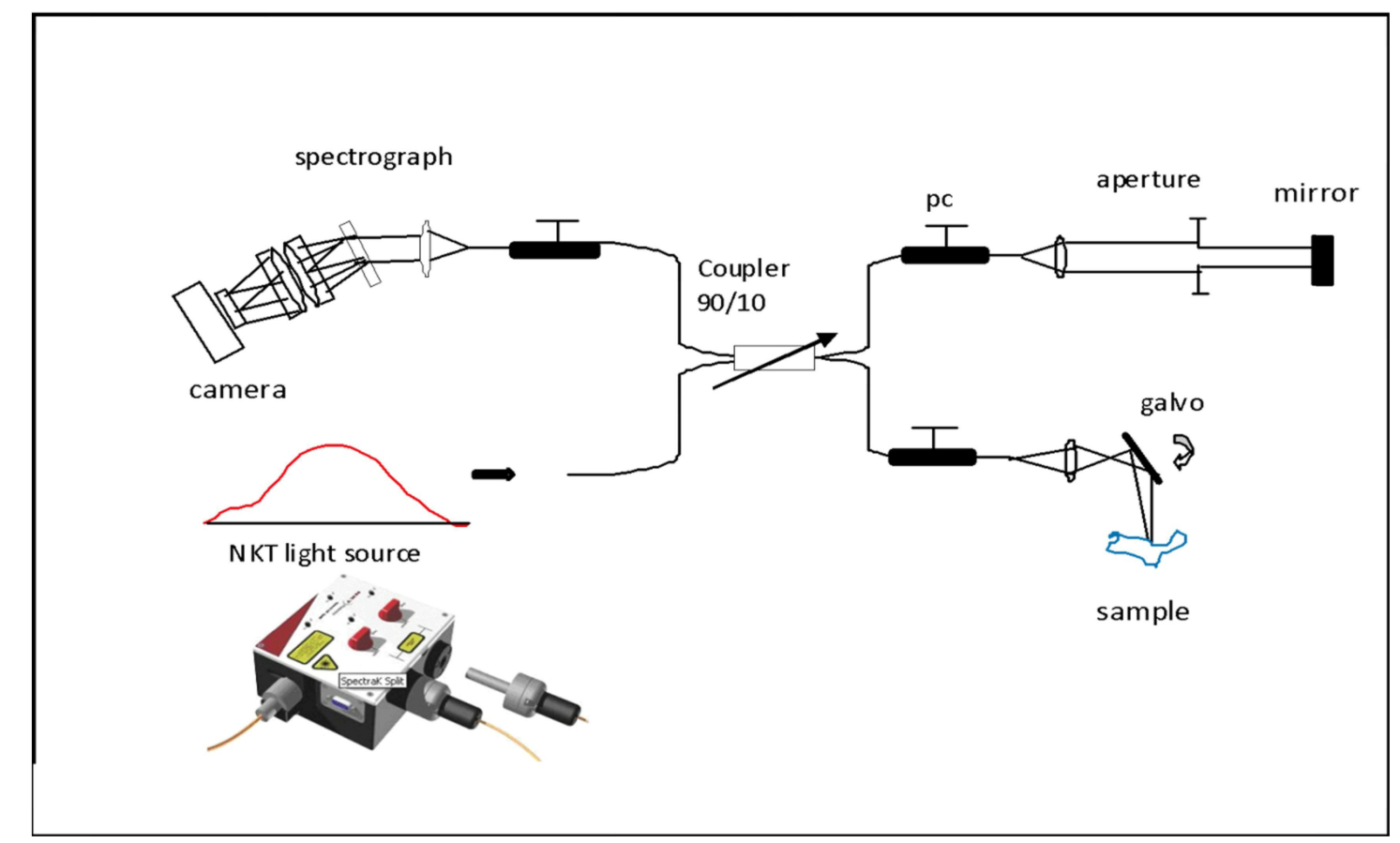

Figure 1. Experimental setup of the ultra-high resolution 810nm spectral domain OCT using the NKT SuperK Versa supercontinuum light source with a 1200 1/mm grating, a 4096 pixels linear CCD detector array.

Figure 1 shows the instrument setup. The broadband light is coupled into the input arm of a $2 \times 2$ fibre coupler with a cutoff wavelength below $600 \mathrm{~nm}$. One of the output arms is used to illuminate a probe with gallvo scanning mirrors which scans and collect light from the sample. The returned light from the sample recombines with the reflected light from the reference mirror and the signal is captured in the spectrograph. The light is dispersed by a transmission grating (Wasatch) 1200 lines/mm and captured on a line CCD camera (E2V) with 4096 pixels (pixel size 10x10 $\mu \mathrm{m}$ ) using a ZEISS $85 \mathrm{~mm}$ focal length camera lens. The whole system is controlled by a personal computer.

The NKT SuperK Versa source spectrum in the $600 \mathrm{~nm}-1000 \mathrm{~nm}$ wavelength region is captured by the spectrograph (central wavelength of $810 \mathrm{~nm}$, FWHM $180 \mathrm{~nm}$ ). Using such a broadband source presents many challenges in the design and in the availability of the optical components. The main challenge is to maintain a broadband while making sure a small focused spot is maintained over the entire length of the linear detector $(4 \mathrm{~cm}$ length) thus ensuring a reasonable depth range $(>1 \mathrm{~mm})$. The optical components, the CCD detector all contributes to the shaping of the spectrum of the injected light.

The system is wavelength calibrated using 20 known arc lines from Neon and Xenon lamps (Ocean Optics). The spectra are then resampled to uniform k-spacing, continuum subtracted and tapered with a Hann window before Fourier transforming to obtain the depth profiles and cross-section images 


\section{RESULTS}

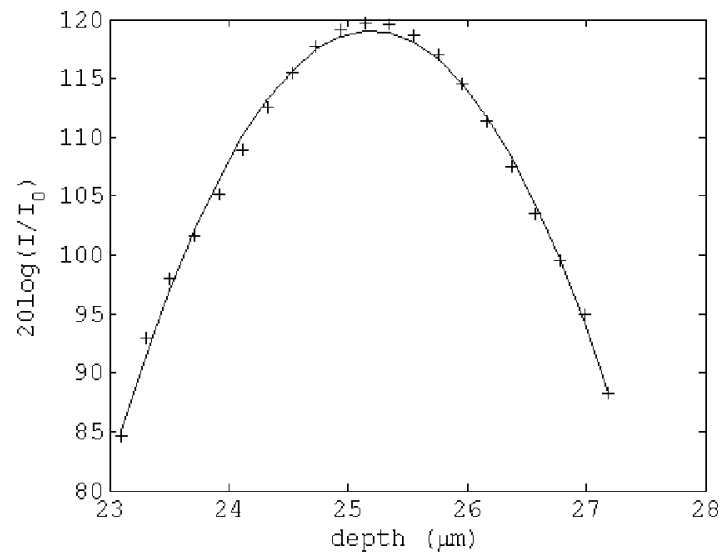

(a)

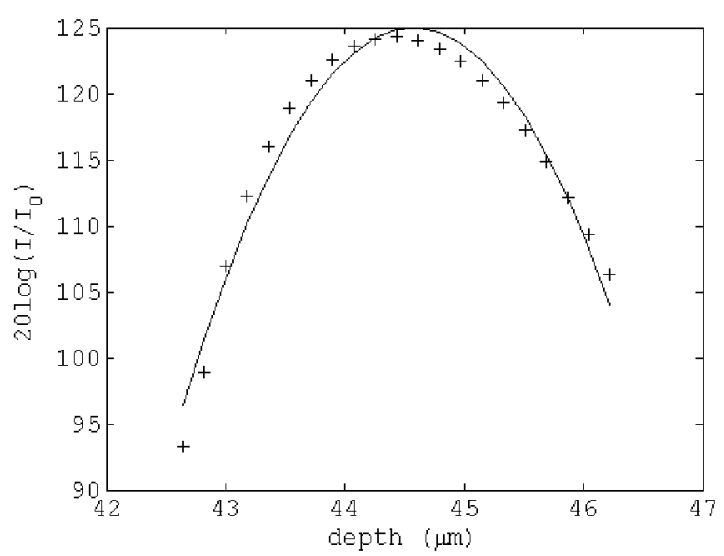

(b)

Figure 2 a) Simulated depth profile of the OCT using a measured NKT source spectrum and the CCD detector spectral response. The simulated spectrum is then processed in the same way as the actual measured data; b) The measured depth profile of a flat glass slide was found to have a FWHM depth resolution of $\sim 1.8 \mu \mathrm{m}$ in air consistent with the simulated value of $1.8 \mu \mathrm{m}$. The red curves are Gaussian fits to the profiles.

The resolution of the system was measured with a glass microscope slide and found to be $\sim 1.8 \mu \mathrm{m}$ in air without any tapering of the spectrum (Fig. 2b), or $\sim 2.2 \mu \mathrm{m}$ in air if tapered with a Hann window. This is found to be consistent with the theoretically expected resolution taking into account the CCD detector spectral response as well as the actual measured source spectrum (Fig. 2a).

Figure 3 shows cross-section images of a layer of Regalrez varnish on ground glass obtained with the 810nm ultra-high resolution (UHR) OCT and a 930 $\mathrm{nm}$ OCT at a lower resolution $(6.5 \mu \mathrm{m}$ axial resolution).

Figure 4 shows the cross-section image of a madder lake paint in egg tempera obtained with the UHR OCT compared with a lower resolution OCT. The UHR OCT image shows clearly the scattering centers in the paint. The higher resolution also meant that the diffuse multi-scattering features in the low resolution images are not picked up. 


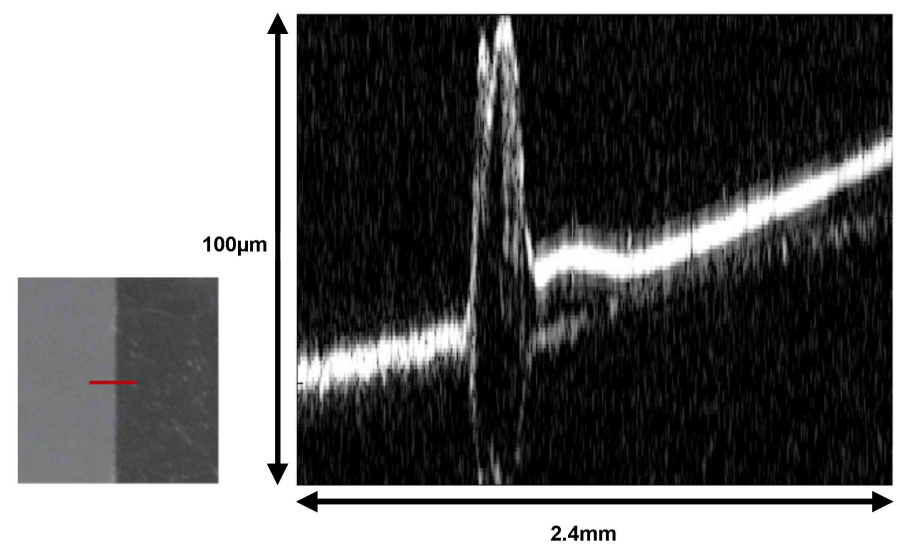

(a)

(b)

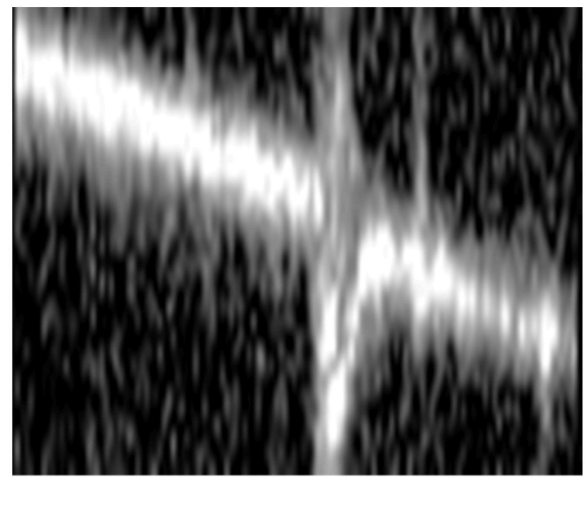

(c)

Figure 3: a) A thin layer of Regalvez varnish on ground glass (left side of image is unvarnished surface); b) cross-section image using the ultra-high resolution OCT at $810 \mathrm{~nm}$ along the red line segment shown in a); c) with a FD-OCT at 930nm (depth resolution $\sim 6.5 \mu \mathrm{m})$ at a similar region. The two images are on the same scale in both dimensions $(2.4 \mathrm{~mm}$ wide and $0.1 \mathrm{~mm}$ depth).

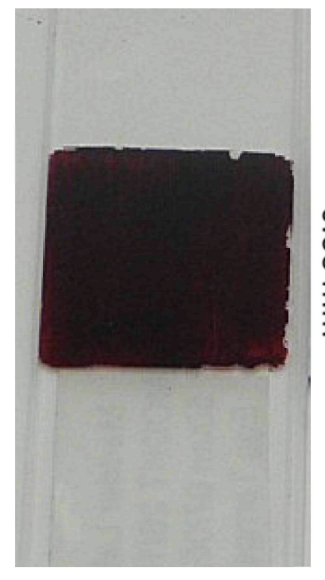

(a)

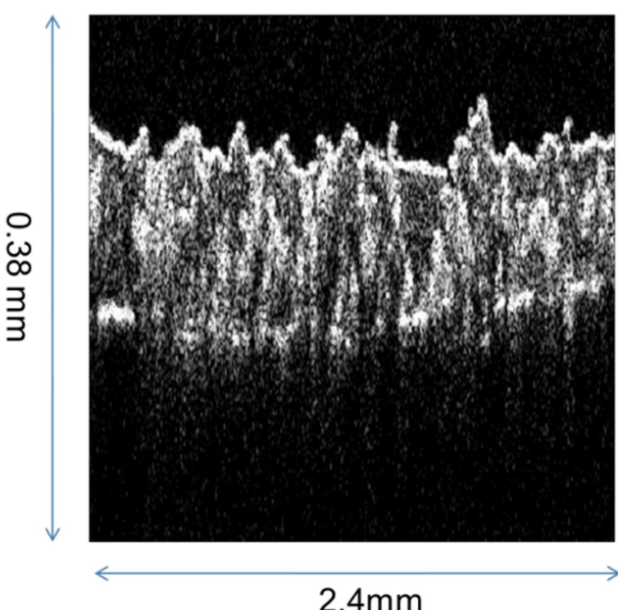

(b)

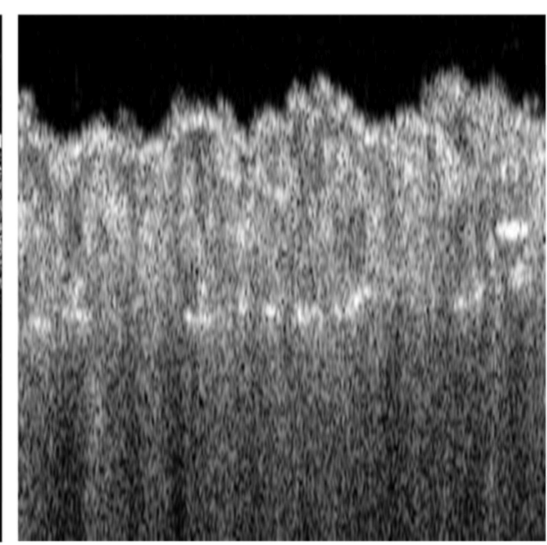

(c)

Figure 4: a) Madder lake in egg tempera painted on a glass microscope slide; b) cross-section image with the ultra-high resolution OCT at $810 \mathrm{~nm}$ and c) cross-section image with an OCT at $930 \mathrm{~nm}$ (depth resolution $\sim 6.5 \mathrm{~mm}$ ) at a similar region. The two images are on the same scale in both dimensions.

\section{CONCLUSION}

By using a supercontinuum source, we have developed an ultra-high resolution spectral domain OCT at $\sim 810 \mathrm{~nm}$ for high depth resolution imaging of varnish and paint layers. The theoretical depth resolution of $1.8 \mu \mathrm{m}$ (in air) is achieved and it is shown to be able to resolve thin varnish and paint layers. 


\section{ACKNOWLEDGEMENTS}

Funding from UK AHRC and EPSRC Science \& Heritage Programme (Interdisciplinary Research Grant AH/H032665/1) is gratefully acknowledged. Paint samples are provided by the National Gallery.

\section{REFERENCES}

[1] Targowski, P., Iwanicka, M., "Optical Coherence Tomography for structural examination of cultural heritage objects and monitoring of restoration processes - a review," Applied Physics A 106(2), 265-277 (2012).

[2] Liang, H., Peric B., Hughes M., Podoleanu A., Spring M., Roehrs S., "Optical Coherence Tomography in Archaeology and Conservation Science - A new emerging field," Proc. SPIE 7139, 713915 (2008).

[3] Yang, M. L., Lu, C.W., Yang, C. C., "The use of optical coherence tomography for monitoring the subsurface morphology of archaic jades," Archaeometry 46, 171-182 (2004).

[4] Liang, H.,Sax, M.; Saunders, D. and Tite, M., "Optical Coherence Tomography for the non-invasive investigation of the microstructure of ancient Egyptian faience," Journal of Archaeological Science 39, 36833690 (2012).

[5] Lawman, S. and Liang, H., "High precision dynamic multi-interface profilometry with optical coherence tomography," Applied Optics 50(32), 6039-6048 (2011).

[6] Liang, H., Cid, M., Cucu, R., Dobre, G., Podoleanu, A., Pedro, J., Saunders, D., "En-face optical coherence tomography - a novel application of non-invasive imaging to art conservation," Opt. Express 13, 6133-6144 (2005).

[7] Latour, G., Echard, J., Soulier, B., Emond, I., Vaiedelich, S., Elias, M., "Structural and optical properties of wood and wood finishes studied using optical coherence tomography: application to an 18th century Italian violin," Applied Optics 48, 6485-6491 (2009).

[8] Gurov, I., Karpets, A., Margariants, N., Vorobeva, E., "Full-field high-speed optical coherence tomography system for evaluating multilayer and random tissues," Proc. SPIE 6618, 661807 (2007). 